\section{Diabetes, Obesity, and Acrp30/Adiponectin}

\author{
Christopher Hug ${ }^{1,2}$ and Harvey F. Lodish ${ }^{1,3}$ \\ ${ }^{1}$ Whitehead Institute for Biomedical Research, \\ Cambridge, ${ }^{2}$ Children's Hospital, Boston, and \\ ${ }^{3}$ Massachusetts Institute of Technology, Cam- \\ bridge, MA, USA
}

Diabetes mellitus (DM) is the clinical condition of inappropriate regulation of blood sugar by insulin, with resulting elevation of blood glucose and several other metabolic derangements. DM is comprised of two distinct entities: DM type 1 (DM-1), affecting the minority (5\%-10\%), is characterized by the destruction of insulin-secreting pancreatic $\beta$ cells, leading to an absence of insulin. DM type 2 (DM-2), also called adult-onset DM and affecting $90 \%-95 \%$ of patients with diabetes, is characterized by peripheral defects in the response to insulin. Much work has focused on the underlying pathophysiology of these two diseases, related by a common metabolic defect-an absolute or functional loss of insulin action-but differing widely in pathogenesis. [For a recent review, see Saltiel (25)]. Insulin, as an anabolic hormone, acts to promote the clearance of glucose, chiefly in muscle through the stimulation of translocation of GLUT4 from intracellular pools to the plasma membrane; in liver by the inhibition of glycogenolysis and the enhancement of glycogen synthesis; and in adipose tissue by the uptake of free fatty acids (30) and its storage as fat. In adipose tissue, insulin also inhibits the breakdown of triglycerides and the resultant release of fatty acids by inhibiting hormone-sensitive lipase.

The prevalence of DM-2 has continued to increase in recent years, with the number of patients in selected areas of the United States increasing 3-fold over an eight-year period (3), and is matched by a striking rise in childhood DM-2 (13). This increase has paralleled an increase in obesity, highlighting the intimate connection of these conditions. This link has been described in both the developed and increasingly in the developing world, demonstrating the influence of lifestyle on pathogenesis. However, strong genetic links between obesity and diabetes have also been described, and these links have been supported at the basic science level as well. In particular, derangements in lipid metabolism are a hallmark of DM-2. Much of the morbidity associated with obesity may be directly or indirectly associated with diabetes, as poorly controlled diabetes and obesity lead to a constellation of symptoms that are together known as the metabolic syndrome, or syndrome X $(7,11,12)$. The term "the deadly quartet", coined by Kaplan (11), refers to the abdominal obesity, insulin resistance, dyslipidemia, and hypertension found in the metabolic syndrome. Clearly, there are many factors underlying the development and progression of these two related conditions, and developing methods to counter them has become a major goal of contemporary medicine.

Recent work has shown that adipose tissue, while long known for its capacity to store fat, has an additional important role as the source for a number of hormones and paracrine mediators, including resistin, adipsin, leptin, and TNF- $\alpha$. Many groups are studying the physiologic role of these molecules and their connection to DM-2. Here we discuss a recently identified hormone produced only by adipocytes, Acrp30 (also called adiponectin), that may be central to the development of DM-2 and the metabolic syndrome.

Acrp30 (adipocyte complement-related protein of 30 $\mathrm{kDa}$ ) is a serum protein produced exclusively by adipose tissue. First identified in 1995 (28) in a subtractive hybridization screen to identify genes induced during differentiation of mouse 3T3-L1 preadipocytes to adipocytes, Acrp30 is up-regulated over 100-fold during adipocyte differentiation. Subsequently, other groups have identified the human homolog, called Apm1 (20).

The predicted amino acid sequence (Figure 1) contains an amino-terminal signal sequence, followed by a nonconserved region of 28 amino acids, 22 collagen repeats, and a carboxyl-terminal domain with homology to the globular complement factor C1q. This segment is also homologous to Hib27, a member of a family of proteins found in the serum of animals capable of hibernation, but only when the animals are in the active state $(15,28)$. Bacterially expressed full-length protein forms trimers, hexamers, and higher-order structures (31); similar species are also seen in serum, where the protein is found at high concentration, on the order of $5-10 \mu \mathrm{g} / \mathrm{mL}(1,28)$. The significance of these different species is further described below.

The crystal structure of the globular head alone, expressed in bacteria, has similarity to TNF- $\alpha$ (29), forming a homotrimer with characteristic topology; the amino terminal and carboxyl termini are located in close proximity. Post-translational modifications include the addition of disialic acid residues (26) and hydroxylation and glycosylation at conserved lysine residues in the amino-terminal region of the molecule. Though the functional signifi-

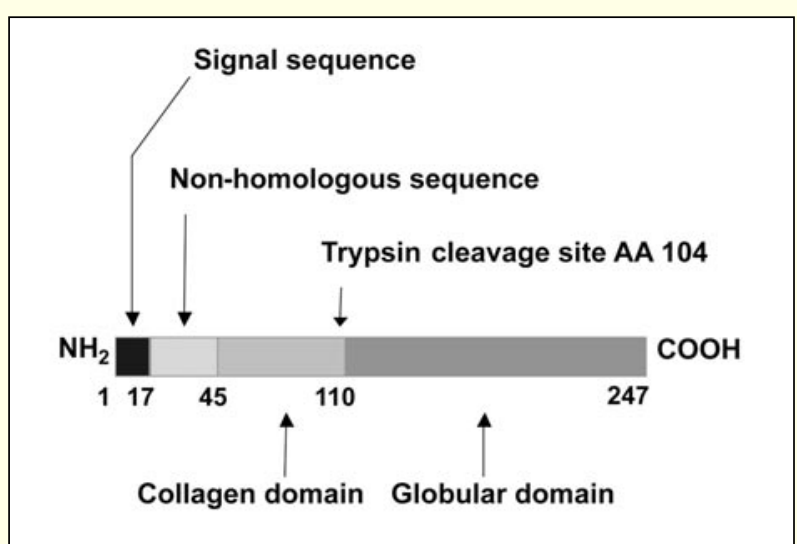

Figure 1. The schematic structure of Acrp30 and gAcrp30 is shown together with several of its structural features. The mature molecule found in serum is a homotrimer of three identical subunits that in turn assemble into the higher-ordered structures discussed in the text. 
cance is unclear, the latter may relate to the stabilization of the collagen domain (32).

Evidence for the in vivo activity of Acrp30 relating to energy metabolism has emerged from several lines of research. First, genetic data has demonstrated linkage of DM-2 with non-coding SNPs located within the Acrp30 gene in a Japanese cohort of patients (6), and missense mutations affecting the globular head are correlated with serum levels of Acrp30 (14). Second, serum levels of Acrp30 are decreased in several models of obesity, including leptin-deficient mice (10), leptin-receptor-deficient mice (33), monkey (9), and in human obesity and in DM-2. In human studies, Acrp30 levels are negatively correlated with both diabetes and obesity and are further reduced in patients with coronary artery disease (1).

That exogenous Acrp30 might function as a therapeutic was first explored by Fruebis et al. (5), in which injection of the carboxyl-terminal globular domain (gAcrp30; Figure 1), prepared by trypsin digestion of bacterially produced full-length protein, had potent effects in inducing sustained weight loss in obese mice fed a high-fat diet. In these studies, the effect of full-length Acrp30 was less marked, supporting the hypothesis that the full-length molecule is converted to a more active globular form, possibly by enzymatic action. Indeed, a carboxyl-terminal fragment of the molecule was found in serum using globular-domain-specific antibodies, albeit at low concentration. This finding has been confirmed by others (33).

Gene disruption experiments yielded supporting results, although a slightly different phenotype was seen by two groups, and a third group (19) found increased $\beta$ oxidation but no other metabolic effects resulting from the deletion of the Acrp30 gene. Kubota et al. (16) observed moderate insulin resistance in mice that were heterozygous for the Acrp30 gene; this effect was more severe in mice lacking both copies of the gene. The two types of mice had body weights similar to wild-type mice. When the femoral artery of homozygous mice was injured with a balloon cuff, there was a 2-fold increase in neointimal thickening, suggesting a role for Acrp30 in maintaining the integrity of the vessel wall, a function first suggest by Matsuzawa et al. (23).

Maeda et al. (21) generated a similar mouse strain; however, these homozygous Acrp30-deficient mice were not hyperglycemic when maintained on a normal diet, although they did exhibit reduced clearance of serum-free fatty acid. When switched to a high-fat, high-sucrose diet, they exhibited severe insulin resistance and demonstrated increased weight gain relative to control animals. At baseline, TNF- $\alpha$ was increased in serum and adipose tissue of the homozygous mice, and skeletal muscle expression of FATP-1, a transmembrane protein involved in cellular uptake of serum fatty acids $(27,30)$, was decreased. These biochemical alterations were corrected by infecting the mice with an adenoviral vector expressing Acrp30. These data suggest that Acrp30 and TNF- $\alpha$ act in counter-regulatory pathways and that the net balance of their actions results in the proper homeostasis of glucose and fatty acid metabolism. Under conditions of 
metabolic stress, such as diet-induced obesity in the Acrp30 mutant, unopposed TNF- $\alpha$ activity may lead to a shift toward insulin resistance.

The reduced levels of Acrp30 seen in a variety of insulin-resistant states, such as humans with DM-2 or obesity, and mice with a variety of genetically induced obesity and diabetic states, are corrected by several therapies, including weight loss, caloric restriction, and treatment with PPAR- $\gamma$ agonists $(4,22)$. However, the specific mechanism of action of Acrp30, induced either directly by injection of recombinant protein or viral infection or indirectly by PPAR- $\gamma$ agonists or weight loss, is unknown.

Acrp30 is present in serum at high concentration $(5-10 \mu \mathrm{g} / \mathrm{mL})$ and exists as multiple pools of different apparent molecular weight, as determined by sucrose density centrifugation and chemical cross-linking studies (28). Additionally, a polypeptide lacking the amino-terminus of the protein exits in serum $(5,33)$, although this observation has not been confirmed by all investigators (32). This raises the question as to the identity of the active form(s) of this protein.

To address this, Tsao et al. (31) examined the relative distribution of several preparations of Acrp30. When expressed in bacteria as a full-length fusion protein and separated by gel-filtration chromatography, three forms of
Acrp30 were identified, corresponding to hexamer, trimer $\mathrm{A}$, and trimer B; trimer B was an unfolded artifact of bacterial expression. Eukaryotic cell expression studies generated three Acrp30 species: a high molecular weight species (not seen in bacterially produced protein) and species corresponding to hexamer and Trimer A. Mouse serum contained Acrp30 species similar to those made by recombinant expression in eukaryotic cells.

In contrast, similar studies on gAcrp30 (produced in bacteria) indicate that it exists predominantly as a homotrimer and does not form significant amounts of higher-order structures.

To determine the potential activity of these different forms, a number of transcriptional response elements driving the expression of a luciferase reporter gene were assayed in $\mathrm{C} 2 \mathrm{C} 12$ myocytes, cells known to respond to Acrp30. The NF- $\mathrm{KB}$-responsive E-selectin promoter demonstrated increased activity in response to the addition of bacterially produced Acrp30; this was associated with degradation via phosphorylation of the inhibitory protein IKB- $\alpha$. Only the fractionated hexamer and larger species were active. Interestingly, gAcrp30 was inactive in this assay.

Several structural aspects of Acrp30 may explain these results. First, the valency of the globular heads may be increased by the oligomerization of Acrp30; such increased valency would not be possible with gAcrp30, which may therefore bind cell surface receptors with lower affinity than Acrp30. Second, Acrp30 and gAcrp30 may bind distinct cell surface receptor proteins and/or activate distinct intracellular signal transduction pathways. Future experiments should distinguish between these possibilities.

There are differences in the activities of Acrp30 and gAcrp30 in vivo, although the two molecules share similarities as well. When injected into mice, gAcrp30 is more potent in ameliorating the rise in serum glucose following a glucose challenge and in promoting clearance of free fatty acids. Following long-term treatment with recombinant protein, there was a greater reduction in weight with injection of gAcrp30 compared to Acrp30 (5), but no difference in food intake. In this study, gAcrp30, applied to isolated muscle tissue or differentiated C2C12 muscle cells, increased oleate oxidation relative to control cells in which buffer was applied.

Scherer et al. (28) studied the effects of Acrp30, prepared in a eukaryotic cell expression system, on isolated hepatocytes. Acrp30 reduced glucose production synergistically with insulin, which was required for the action of Acrp30 (2). Bacterially produced gAcrp30 had no activity in this assay. Further experiments are needed to determine whether this finding reflects an inherent difference in the activity between Acrp30 and gAcrp30 or whether these differences relate to eukaryotic cell posttranslational modifications necessary for certain Acrp30 activities but lacking in bacterially expressed proteins

Thus, the major sites of action of Acrp30/gAcrp30 appear to be muscle and liver, as there have been no studies describing the effects of Acrp30 on metabolism of adipocytes or other types of cells. 
TNF- $\alpha$ induces insulin resistance and has been implicated in the development of DM-2, although a causal role in development of the disease has not been established and the mechanisms by which TNF- $\alpha$ exerts its effects are unknown. TNF- $\alpha$ levels are elevated in diabetes and obesity (8), and metabolic syndrome is associated with increased levels of systemic inflammatory cytokines (17) as well.

Ruan et al. (24) demonstrated that treatment of 3T3L1 adipocytes with TNF- $\alpha$ represses the expression of many adipocyte-specific genes, including Acrp30, and induces the expression of many inflammatory chemokines and cytokines. The overall pattern of gene expression becomes similar to that of pre-adipocytes, suggesting that TNF- $\alpha$ induces a state of adipocyte de-differentiation. These effects of TNF- $\alpha$ required signaling through NF$\kappa \mathrm{B}$, as adipocytes expressing a non-degradable inhibitor of NF- $\kappa B$ did not exhibit most of these alterations in gene expression following treatment with TNF- $\alpha$. Thus, part of the mechanism by which TNF- $\alpha$ induces insulin resistance could involve a reduction in Acrp30 production.

As described above, mice lacking Acrp30 through homologous recombination have elevated levels of TNF- $\alpha$, which is reversed by expressing Acrp30; however, the mechanism of Acrp30's action on TNF- $\alpha$ is unknown and may reflect a direct or indirect effect. In either case, this supports a model of pro- and anti-TNF- $\alpha$ effects in which Acrp30 acts to counteract both the actions and levels of TNF- $\alpha$. Were this the case, one might expect alterations in TNF- $\alpha$ and/or Acrp30 levels before the development of frank glucose intolerance. Indeed, this is seen in the Acrp30-deficient mice, which have normal baseline glucose and free fatty acid levels yet exhibit abnormal responses when stressed by the infusion of free fatty acids or diet-induced obesity. Supporting a causal relationship between reduced levels of Acrp30 and the development of insulin resistance and diabetes, in the Pima Indian population, individuals who later developed DM-2 had lower serum levels of Acrp30 than those who did not (18).

DM-2 and obesity, combining with the later development of the metabolic syndrome, are clearly diseases of the Western and developing worlds, with strong influences of genetics and lifestyle. To combat the human and financial costs, drugs that act to reverse the effects or likelihood of developing DM-2 must be developed, and Acrp30 and its fragment gAcrp30 are candidate molecules. A further understanding of the pathways through which these molecules act may open new avenues for pharmacological intervention. 


\section{ACKNOWLEDGMENTS}

We thank Drs. J. Bogan and T.-S. Tsao for valuable discussions. C.H. is supported by a training grant from the National Institutes of Health through the Division of Respiratory Diseases, Children's Hospital Boston. Work on Acrp30 in H.F.L's laboratory is supported by National Institutes of Health grant no. R37DK47618 and by a grant from Genset.

\section{REFERENCES}

1.Arita, Y., S. Kihara, N. Ouchi, M. Takahashi, K. Maeda, J. Miyagawa, K. Hotta, I. Shimomura, et al. 1999. Paradoxical decrease of an adipose-specific protein, adiponectin, in obesity. Biochem. Biophys. Res. Commun. 257:79-83.

2.Berg, A.H., T.P. Combs, X. Du, M. Brownlee, and P.E. Scherer. 2001. The adipocyte-secreted protein Acrp30 enhances hepatic insulin action. Nat. Med. 7:947-953.

3.Burke, J.P., K. Williams, S.P. Gaskill, H.P. Hazuda, S.M. Haffner, and M.P. Stern. 1999. Rapid rise in the incidence of type 2 diabetes from 1987 to 1996: results from the San Antonio Heart Study. Arch. Intern. Med. 159:1450-1456.

4.Combs, T.P., J.A. Wagner, J. Berger, T. Doebber, W.J. Wang, B.B. Zhang, M. Tanen, A.H. Berg, et al. 2002. Induction of adipocyte complement-related protein of 30 kilodaltons by PPARgamma agonists: a potential mechanism of insulin sensitization. Endocrinology 143:998-1007.

5.Fruebis, J., T.S. Tsao, S. Javorschi, D. Ebbets-Reed, M.R. Erickson, F.T. Yen, B.E. Bihain, and H.F. Lodish. 2001. Proteolytic cleavage product of $30-\mathrm{kDa}$ adipocyte complement-related protein increases fatty acid oxidation in muscle and causes weight loss in mice. Proc. Natl. Acad. Sci. USA 98:2005-2010.

6.Hara, K., P. Boutin, Y. Mori, K. Tobe, C. Dina, K. Yasuda, T. Yamauchi, S. Otabe, et al. 2002. Genetic variation in the gene encoding adiponectin is associated with an increased risk of type 2 diabetes in the Japanese population. Diabetes 51:536-540.

7.Hauner, H. 2002. Insulin resistance and the metabolic syndrome-a challenge of the new millennium. Eur. J. Clin. Nutr. 56(Suppl 1):S25-S29.

8.Hotamisligil, G.S., P. Arner, J.F. Caro, R.L. Atkinson, and B.M. Spiegelman. 1995. Increased adipose tissue expression of tumor necrosis factor-alpha in human obesity and insulin resistance. J. Clin. Invest. 95:2409-2415.

9.Hotta, K., T. Funahashi, N.L. Bodkin, H.K. Ortmeyer, Y. Arita, B.C. Hansen, and Y. Matsuzawa. 2001. Circulating concentrations of the adipocyte protein adiponectin are decreased in parallel with reduced insulin sensitivity during the progression to type 2 diabetes in rhesus monkeys. Diabetes 50:1126-1133.

10.Hu, E., P. Liang, and B.M. Spiegelman. 1996. AdipoQ is a novel adipose-specific gene dysregulated in obesity. J. Biol. Chem. 271:10697-10703.

11.Kaplan, N.M. 1989. The deadly quartet. Upper-body obesity, glucose intolerance, hypertriglyceridemia, and hypertension. Arch. Intern. Med. 149:1514-1520.

12.Kaplan, N.M. 1998. Obesity in hypertension: effects on prognosis and treatment. J. Hypertens. Suppl. 16:S35-S37.

13.Kaufman, F.R. 2002. Type 2 diabetes mellitus in children and youth: a new epidemic. J. Pediatr. Endocrinol. Metab. 15(Suppl 2):737-744.

14.Kondo, H., I. Shimomura, Y. Matsukawa, M. Kumada, M. Takahashi, M. Matsuda, N. Ouchi, S. Kihara, et al. 2002. Association of adiponectin mutation with type 2 diabetes: a candidate gene for the insulin resistance syndrome. Diabetes 51:2325-2328.

15.Kondo, N. and J. Kondo. 1992. Identification of novel blood proteins specific for mammalian hibernation. J. Biol. Chem. 267:473478.

16.Kubota, N., Y. Terauchi, T. Yamauchi, T. Kubota, M. Moroi, J. Matsui, K. Eto, T. Yamashita, et al. 2002. Disruption of adiponectin causes insulin resistance and neointimal formation. J. Biol. Chem. 277:25863-25866.

17.Lin, Y., M.W. Rajala, J.P. Berger, D.E. Moller, N. Barzilai, and P.E. Scherer. 2001. Hyperglycemia-induced production of acute phase reactants in adipose tissue. J. Biol. Chem. 276:42077-42083.

18.Lindsay, R.S., T. Funahashi, R.L. Hanson, Y. Matsuzawa, S. Tanaka, P.A. Tataranni, W.C. Knowler, and J. Krakoff. 2002. Adiponectin and development of type 2 diabetes in the Pima Indian population. Lancet 360:57-58.

19.Ma, K., A. Cabrero, P.K. Saha, H. Kojima, L. Li, B.H. Chang, A. Paul, and L. Chan. 2002. Increased beta-oxidation but no insulin resistance or glucose intolerance in mice lacking adiponectin. J. Biol. Chem. 31:31.

20.Maeda, K., K. Okubo, I. Shimomura, T. Funahashi, Y. Matsuzawa, and K. Matsubara. 1996. cDNA cloning and expression of a novel adipose specific collagen-like factor, apM1 (AdiPose Most abundant Gene transcript 1). Biochem. Biophys. Res. Commun. 221:286-289.

21.Maeda, N., I. Shimomura, K. Kishida, H. Nishizawa, M. Matsuda, H. Nagaretani, N. Furuyama, H. Kondo, et al. 2002. Diet-induced insulin resistance in mice lacking adiponectin/ACRP30. Nat. Med. 8:731-737.

22.Maeda, N., M. Takahashi, T. Funahashi, S. Kihara, H. Nishizawa, K. Kishida, H. Nagaretani, M. Matsuda, et al. 2001. PPARgamma ligands increase expression and plasma concentrations of adiponectin, an adipose-derived protein. Diabetes 50:2094-2099.

23.Ouchi, N., S. Kihara, Y. Arita, K. Maeda, H. Kuriyama, Y. Okamoto, K. Hotta, M. Nishida, et al. 1999. Novel modulator for endothelial adhesion molecules: adipocyte-derived plasma protein adiponectin. Circulation 100:2473-2476.

24.Ruan, H., N. Hacohen, T.R. Golub, L. Van Parijs, and H.F. Lodish. 2002. Tumor necrosis factor-alpha suppresses adipocytespecific genes and activates expression of preadipocyte genes in 3T3-L1 adipocytes: nuclear factor-kappaB activation by TNF-alpha is obligatory. Diabetes 51:1319-1336.

25.Saltiel, A.R. 2001. New perspectives into the molecular pathogenesis and treatment of type 2 diabetes. Cell 104:517-529.

26.Sato, C., Z. Yasukawa, N. Honda, T. Matsuda, and K. Kitajima. 2001. Identification and adipocyte differentiation-dependent expression of the unique disialic acid residue in an adipose tissue specific glycoprotein, adipo Q. J. Biol. Chem. 29:29.

27.Schaffer, J.E. and H.F. Lodish. 1994. Expression cloning and characterization of a novel adipocyte long chain fatty acid transport protein. Cell 79:427-436

28.Scherer, P.E., S. Williams, M. Fogliano, G. Baldini, and H.F. Lodish. 1995. A novel serum protein similar to C1q, produced exclusively in adipocytes. J. Biol. Chem. 270:26746-26749.

29.Shapiro, L. and P.E. Scherer. 1998. The crystal structure of a complement-1q family protein suggests an evolutionary link to tumor necrosis factor. Curr. Biol. 8:335-338.

30.Stahl, A., J.G. Evans, S. Pattel, D. Hirsch, and H.F. Lodish. 2002. Insulin causes fatty acid transport protein translocation and enhanced fatty acid uptake in adipocytes. Dev. Cell. 2:477-488.

31.Tsao, T. S., H. E. Murrey, C. Hug, D. H. Lee, and H. F. Lodish. 2002. Oligomerization state-dependent activation of NF-kB signaling pathway by Acrp30. J Biol Chem. 26:26.

32.Wang, Y., A. Xu, C. Knight, L.Y. Xu, and G.J. Cooper. 2002. Hydroxylation and glycosylation of the four conserved lysine residues in the collagenous domain of adiponectin. Potential role in the modulation of its insulin-sensitizing activity. J. Biol. Chem. 23:23.

33.Yamauchi, T., J. Kamon, H. Waki, Y. Terauchi, N. Kubota, K. Hara, Y. Mori, T. Ide, et al. 2001. The fat-derived hormone adiponectin reverses insulin resistance associated with both lipoatrophy and obesity. Nat. Med. 7:941-946.

Address correspondence to Dr. Harvey F. Lodish, Whitehead Institute for Biomedical Research, 9 Cambridge Center, Cambridge, MA 02142,USA.e-mail: lodish@wi.mit.edu 